\title{
Metformin Induces Autophagy via the AMPK-mTOR Signaling Pathway in Human Hepatocellular Carcinoma Cells
}

This article was published in the following Dove Press journal: Cancer Management and Research

\author{
Chun Gao (D) \\ Long Fang' \\ Hui Zhang ${(\mathbb{D})^{2}}^{2}$ \\ Wei-Shuo Zhang (D) \\ Xiao-Ou Li' \\ Shi-Yu Du (D) \\ 'Department of Gastroenterology, \\ China-Japan Friendship Hospital, Beijing \\ I00029, People's Republic of China; \\ ${ }^{2}$ Department of Gastroenterology, \\ Beijing Tiantan Hospital, Capital Medical \\ University, Beijing 100070, People's \\ Republic of China
}

Correspondence: Shi-Yu Du Department of Gastroenterology, ChinaJapan Friendship Hospital, Ministry of Health, No. 2 Yinghua East Road, Beijing 100029, People's Republic of China $\mathrm{Tel} / \mathrm{Fax}+86-10-84205503$

Email dushiyu@zryhyy.com.cn
Background: Metformin may exert the anticancer effect on multiple types of cancers and some potential mechanisms have been suggested. Our study was designed to determine the effect of metformin on the cell autophagy and autophagic flux via the AMPK-mTOR signaling pathway in human hepatocellular carcinoma (HCC) cells.

Methods: MHCC97H and HepG2 cell lines were cultured and treated without and with metformin at various concentrations $(2,5,10$ and $20 \mathrm{mM})$ for $48 \mathrm{~h}$. Then, $10 \mathrm{mM}$ was determined as the optimal concentration and the $\mathrm{HCC}$ cells were treated with metformin for $12,24,48$, and $72 \mathrm{~h}$. MTT assay was used to assess the cell viability and Western blotting was used to determine the expression of proteins (LC3-II, p62, phospho-AMPK $\alpha$, phosphomTOR, mTOR, phospho-p70 S6 Kinase, p70 S6 Kinase, PARP1, Caspase-9 and Caspase-3). Autophagy inhibitor 3-methyladenine, EGFP-LC3 and mCherry-GFP-LC3B plasmid transfection were used for further study.

Results: Metformin inhibited significantly the viability of MHCC97H and HepG2 cells in a dose- and time-dependent manner. For the apoptotic properties, activation of Caspase- 9 and Caspase-3 and PARP cleavage were not observed after treatment with metformin. MHCC97H cells were transfected with a EGFP-LC3 plasmid and treatment with metformin could lead to the increased level of LC3-II and decreased level of p62. In metformin-induced autophagy, AMPK expression was activated, and the phosphorylation levels of mTOR and p70 S6 Kinase were inhibited. Metformin treatment and mCherry-GFP-LC3B plasmid transfection showed that metformin could induce the autophagic flux. 3-Methyladenine (3-MA) partly abolished this effect.

Conclusion: Metformin could induce the autophagy, autophagic flux, and activate the AMPK-mTOR signaling pathway in human HCC cells.

Keywords: metformin, hepatocellular carcinoma, autophagy, autophagic flux, AMPK-mTOR signaling pathway

\section{Introduction}

Hepatocellular carcinoma (HCC), one of the common malignancies of digestive system, is the third most common cause of cancer death worldwide. ${ }^{1,2}$ In China, the mounting annual incidence is particularly high and the number is estimated as 40 per 100000 persons per year. ${ }^{3}$ Some definite risk factors for HCC have been identified, including HBV infection, HCV infection, liver cirrhosis, heavy alcohol consumption, and aflatoxin exposure. ${ }^{2,4}$ In recent years, there are abundant evidences showing that diabetes mellitus (DM) is a confirmed risk factor for HCC and 
diabetic patients have a higher incidence of HCC. ${ }^{5,6}$ According to the 8th IDF Atlas, 425 million people have suffered from diabetes in 2007 and the number will rise to 629 million by $2045 .^{7}$

Metformin (1,1-dimethylbiguanide hydrochloride), a biguanide derivative, is one of the most used first-line anti-hyperglycemic drugs for type 2 DM. ${ }^{3}$ Epidemiological studies have demonstrated that treatment with metformin can reduce the risk of some cancers, such as HCC, breast cancer, colorectal cancer and pancreatic cancer. ${ }^{8-10}$ For the association of metformin use with HCC risk, we have published one meta-analysis which included seven studies and 16,549 diabetic patients. ${ }^{3}$ Our overall analysis showed that diabetic patients with metformin use had a significantly reduced risk of $\mathrm{HCC}$ (relative risk 0.24 , 95\% confidence interval 0.13-0.46). These results were supported by experimental studies and multiple potential anti-cancer mechanisms of metformin were proposed, ${ }^{3,8-10}$ including inhibition of cell proliferation and hepatic gluconeogenesis, activation of AMPK, and modulation of microRNAs expression.

Autophagy, a mechanism by which the cells try to survive, plays important biological roles in the initiation and progression of tumors. ${ }^{11}$ The AMP-activated protein kinase (AMPK)-mammalian target of rapamycin (mTOR) signaling pathway is well known to be associated with autophagy and AMPK can promote the initiation of autophagy. ${ }^{12}$ Activation of AMPK can result in the inhibition of mTOR, which is commonly activated in malignant cells. $^{3,12}$ Some studies suggested that metformin might exert the anticancer effect by regulation of the AMPKmTOR signaling pathway. ${ }^{13,14}$ Our study was designed to determine the effect of metformin on the cell autophagy and autophagic flux via the AMPK-mTOR signaling pathway in human HCC cells.

\section{Materials and Methods}

\section{Reagents and Antibodies}

Metformin, PMSF, leupeptin and aprotinin were purchased from Sigma (St. Louis, MO, USA). Acrylamide, methylene bisacrylamide, sodium dodecyl sulfonate (SDS), Tris base, ammonium persulfate and Tween-20 were obtained from Amresco (Solon, OH, USA). Fetal bovine serum and DMEM were purchased from HyClone (Logan, UT, USA). Antibodies against p62, phospho-AMPK $\alpha$ (Thr172), phospho-mTOR (Ser2448), mTOR, phospho-p70 S6 Kinase (Thr421/Ser424), p70 S6 Kinase and $\beta$-actin were obtained from Cell Signaling (Danvers, MA, USA). Antibodies against PARP1, Caspase-9 and Caspase-3 were purchased from Abcam (Cambridge, MA, USA). Antibody against microtubule-associated protein 1 light chain 3 II (LC3-II) was from GeneTex (Irvine, CA, USA). Anti-rabbit/mouse goat immunoglobulin G-horseradish peroxidase (HRP) secondary antibodies were obtained from Santa Cruz (Santa Cruz, CA, USA). PVDF membrane was purchased from Millipore (Billerica, MA, USA). ECL Plus immunoblotting detection kit was from Bio-Rad (Hercules, CA, USA). Other routine reagents were obtained from Zhongshan (Beijing, China).

\section{Human HCC Cells and Culture}

Our research project was approved by the Clinical Research Ethics Committee of China-Japan Friendship hospital. High metastatic human HCC MHCC97H cell lines were purchased from Beijing BiYao Biotech Co., Ltd. (Beijing, China). The human HCC HepG2 cell lines, which had been authenticated by STR profile, were obtained from the Biochemistry Department of the Health Science Center of Peking University and approved by the Clinical Research Ethics Committee of China-Japan Friendship hospital. MHCC97H and HepG2 cell lines were cultured in Dulbecco's modified Eagle's medium (DMEM, 1 g/L glucose) (Gibco BRL, Grand Island, NY, USA) supplemented with $10 \%$ fetal bovine serum, 100 units $/ \mathrm{mL}$ penicillin, and $100 \mu \mathrm{g} / \mathrm{mL}$ streptomycin sulfate, and maintained at $37^{\circ} \mathrm{C}$ in a humidified $5 \% \mathrm{CO}_{2}$ atmosphere.

\section{MTT Assay}

MTT assay was used to assess the cell viability and it was performed according to the manufacturer's instructions. ${ }^{15}$ Briefly, MHCC97H and HepG2 cells in the logarithmic growth phase were isolated and digested with $0.25 \%$ trypsin cell digestive fluid. After a single cell suspension was prepared, the cells were seeded onto 96-well flat-bottom microtiter plates at a density of $6 \times 10^{3}$ cells per well. $\mathrm{HCC}$ cells in the plates were pre-incubated overnight, allowed to adhere, and treated with metformin at various concentrations $(0,2,5,10$ and $20 \mathrm{mM})$ for $48 \mathrm{~h}$ and $10 \mathrm{mM}$ was selected as the optimal concentration. Then, they were treated with metformin $(10 \mathrm{mM})$ for 24,48 , and $72 \mathrm{~h}$. Six wells for each group, zero-adjustment well and control group were also set. After the treatments, $10 \mu \mathrm{L}$ of MTT reagent $(5 \mathrm{mg} / \mathrm{mL})$ was added to each well and the incubation was continued for $4 \mathrm{~h}$. For each well, a total of $150 \mu \mathrm{L}$ DMSO was added and the absorbance value was 
measured spectrophotometrically at a $570 \mathrm{~nm}$ wavelength. All the experiments were performed in triplicate and repeated thrice.

\section{Western Blotting Analysis}

Western blot analysis was used to determine the expression of proteins (LC3-II, p62, phospho-AMPKa, phospho-mTOR, mTOR, phospho-p70 S6 Kinase, p70 S6 Kinase, PARP1, Caspase-9 and Caspase-3). The experiments were repeated thrice. MHCC97H and HepG2 cells were lysed by adding modified RIPA buffer [50 mM Tris-Hcl (pH7.5), 1\% Triton$100,150 \mathrm{mM} \mathrm{NaCl}, 2 \mathrm{mM}$ EDTA, $1 \mathrm{mM}$ sodium vanadate, $50 \mathrm{mM} \mathrm{NaF}, 1 \mathrm{mM}$ PMSF, $1 \mathrm{mg} / \mathrm{mL}$ aprotinin, $1 \mathrm{mM}$ leupeptin]. The supernatant was collected and bicinchoninic acid protein assay was used to determine the concentration of protein samples. Equal amounts $(40 \mu \mathrm{g})$ of protein samples were loaded onto SDS-containing $12 \%$ polyacrylamide gel and transferred onto a PVDF membrane. After blocking with $5 \%$ nonfat dry milk, the membranes were probed with different primary antibodies, including LC3-II (1:1000), p62 (1:1000), PARP1 (1:1000), Caspase-9 (1:1000), Caspase-3 (1:1000), phospho-AMPK $\alpha$ (Thr172) (1:1000), phosphomTOR (Ser2448) (1:1000), mTOR (1:1000), phospho-p70 S6 Kinase (Thr421/Ser424) (1:1000) and p70 S6 Kinase (1:1000). Secondary antibodies were used at a dilution of 1:5000 (anti-mouse) or 1:2000 (anti-rabbit). Antibody against LC3-II was obtained from GeneTex (Irvine, CA, USA). Antibodies against PARP1, Caspase-9 and Caspase-3 were purchased from Abcam (Cambridge, MA, USA). Antibodies against p62, p-AMPK $\alpha$, p-mTOR, mTOR, p-p70 S6Kinase and p70 S6 Kinase were obtained from Cell Signaling (Danvers, MA, USA).

\section{Plasmids Transfection}

To further investigate the effect of metformin on autophagy in $\mathrm{HCC}$, MHCC97H cells at approximately 60-70\% confluence were transfected with a EGFP-LC3 expression plasmid using the Lipofectamine 2000 transfection reagent (Invitrogen, Carlsbad, CA, USA). ${ }^{16}$ After incubation for $24 \mathrm{~h}$, they were treated with metformin and observed. Moreover, MHCC97H cells were transfected with a mCherry-GFP-LC3B expression plasmid to investigate the effect of metformin on the formation of autophagosome and autolysosome, which can reflect the status of autophagic flux in HCC cells.

\section{Confocal Laser Scanning Microscope}

Before observation, HCC cells were fixed with $4 \%$ formaldehyde for $15 \mathrm{~min}$, and cell nuclei were counterstained with DAPI. An Olympus FV500 confocal laser scanning microscope was used to obtain the images. Five nonoverlapping fields were observed and percentages of cells with punctate EGFP-LC3 structures (green) were calculated. For the results of transfection with mCherry-GFPLC3B, yellow fluorescence signals indicate the change of autophagosome and red fluorescence signals represent the change of autolysosome. Both yellow and red signals are increased simultaneously, indicating that autophagic flux is induced, whereas only yellow signals are increased, indicating that autophagic flux is inhibited. All the experiments were repeated thrice.

\section{Results}

\section{Metformin Inhibits Cell Viability and Induces Autophagy in HCC Cells}

Autophagy was associated with cell viability and death. Before we investigated the effect of metformin on cell autophagy, MTT assay was used to assess the cell viability by metformin in HCC MHCC97H and HepG2 cells (Figure 1A and $\mathrm{B})$. MHCC97H and HepG2 cells were treated with metformin at various concentrations $(2,5,10$ and $20 \mathrm{mM})$ for $48 \mathrm{~h}$. As Figure 1A showed, we found that the HCC cell viability could be inhibited significantly. The inhibitory rate was about $50 \%$ when the cells were treated with $10 \mathrm{mM}$ metformin. Then, $10 \mathrm{mM}$ was determined as the optimal concentration and the cells were treated with metformin for 24, 48 and $72 \mathrm{~h}$ (Figure 1B). The results showed that metformin inhibited the HCC cell viability in a time-dependent manner.

Autophagy and apoptosis are two basic physiologic processes to maintain the cellular homeostasis. PARP1, Caspase- 9 and Caspase-3 were detected by Western blot analysis to determine the apoptotic properties of metformin (Figure 1C and D). MHCC97H and HepG2 cells were treated with metformin $(10 \mathrm{mM})$ for $12,24,48$, and $72 \mathrm{~h}$. The positive control is treated with staurosporine. We found that activation of Caspase-9 and Caspase-3 and PARP cleavage was not observed after treatment with metformin.

To determine the effect of metformin on cell autophagy, autophagy-associated protein expression of LC3-II and p62 was detected by Western blot analysis in MHCC97H and HepG2 HCC cells (Figure 2C and E). 
A

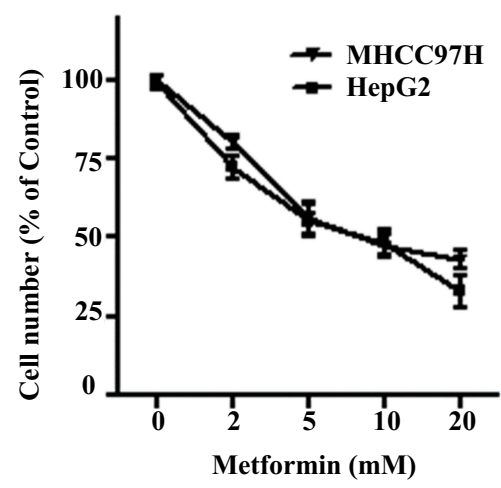

C

MHCC97H $\frac{12 \mathrm{~h}}{\text { C Met }} \frac{24 \mathrm{~h}}{\mathrm{C} \text { Met }} \frac{48 \mathrm{~h}}{\mathrm{C} \text { Met }} \frac{72 \mathrm{~h}}{\mathrm{C} \text { Met STS }}$

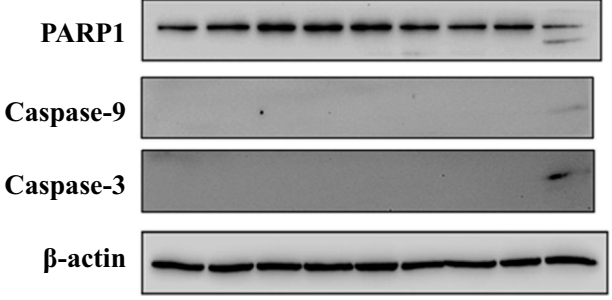

B

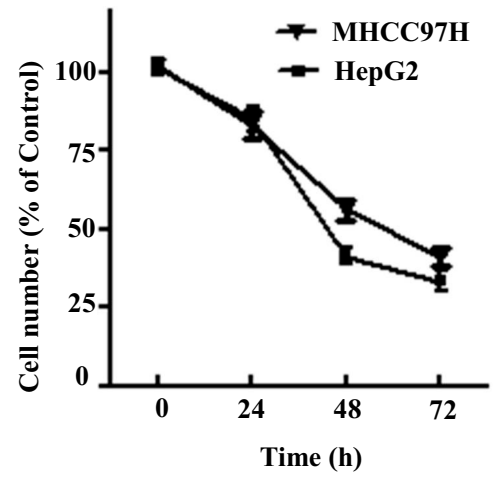

D

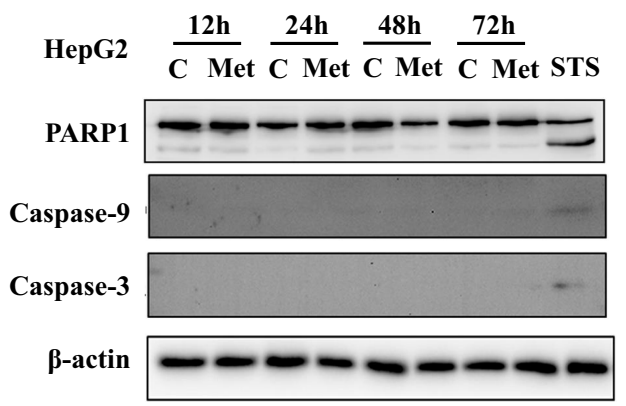

Figure I Metformin inhibits cell viability and the measurement of apoptotic properties of metformin in HCC cells. (A) MHCC97H and HepG2 cells were treated with metformin at various concentrations $(2,5,10$ and $20 \mathrm{mM})$ for $48 \mathrm{~h}$ and $10 \mathrm{mM}$ was selected as the optimal concentration. MTT assay was used to assess the cell viability. (B) MHCC97H and HepG2 cells were treated with metformin (10 mM) for 24, 48, and $72 \mathrm{~h}$. Data were expressed as means \pm standard deviation. (C and $\mathbf{D})$ The apoptotic properties of metformin were measured in MHCC97H and HepG2 HCC cells. PARPI, Caspase-9 and Caspase-3 was detected by Western blot analysis. The "C" indicates the blank control group without treatment, the "Met" indicates the groups treated with metformin, and the "STS" indicates the positive control group which is treated with staurosporine. All the experiments were repeated thrice.

Abbreviations: C, control; h, hour; Met, metformin; STS, staurosporine.

The cells were treated with metformin $(10 \mathrm{mM})$ for 12,24 , 48 , and $72 \mathrm{~h}$. P62 is a substrate of autophagy and it can be degraded by autolysosomes. ${ }^{17}$ Our results found that the p62 protein expression was decreased significantly, indicating that metformin could induce the autophagy in MHCC97H and HepG2 cells. Protein LC3-II is involved in the formation of autophagosomes and serves as one of autophagic markers. ${ }^{17}$ For the expression of LC3-II, we observed that it was increased in MHCC97H cells whereas it was decreased in HepG2 cells (Figure 2C and E). We deduced that metformin might have no obvious effect on the formation of autophagosomes in HepG2 cells, but it could affect any other pathway, such as autophagy flux, which could lead to the decreased LC3-II expression.

\section{EGFP-LC3 Plasmid Transfection and Metformin-Induced Autophagy}

MHCC97H cells were transfected with a EGFP-LC3 plasmid to further investigate the effect of metformin on the formation of autophagosomes (Figure 2A-D). Cells were treated with metformin $(5,10 \mathrm{mM})$ for $48 \mathrm{~h}$ and the results showed that percentages of cells with punctate EGFP-LC3 structures (green fluorescent signals) were increased significantly (Figure 2A and B). Expression of LC3-II and p62 in MHCC97H cells was detected by Western blotting. As shown in Figure 2C and D, treatment with metformin could lead to the increased level of LC3-II expression and decreased level of protein p62 in a dose- and timedependent manner. These results indicated that metformin could induce the formation of autophagosomes and the occurrence of autophagy.

\section{Activation of AMPK in \\ Metformin-Induced Autophagy}

AMPK-mTOR signaling pathway is well known to be associated with cell autophagy and AMPK can promote the initiation of autophagy. ${ }^{12}$ MHCC97H cells were treated with metformin $(5,10$ and $20 \mathrm{mM}$ ) for 24 and $48 \mathrm{~h}$ (Figure 3A). The phosphorylation level of AMPK (Thr172) was analyzed by Western blot. We observed that, compared with the control, expression level of p-AMPKa (Thr172) was increased 
A

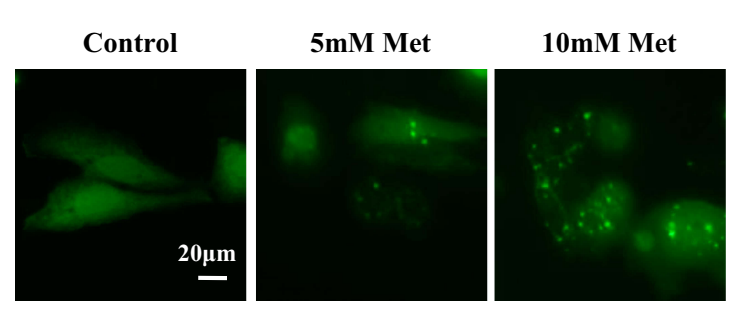

C

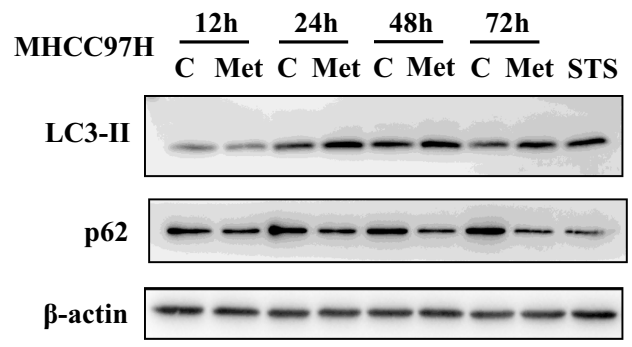

B

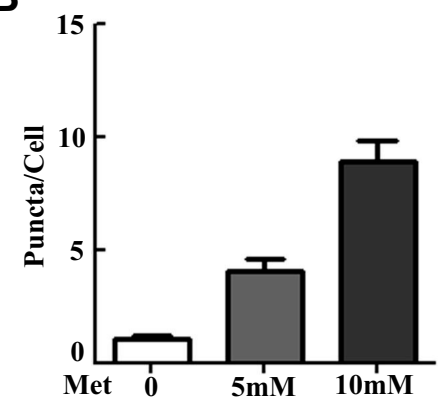

D

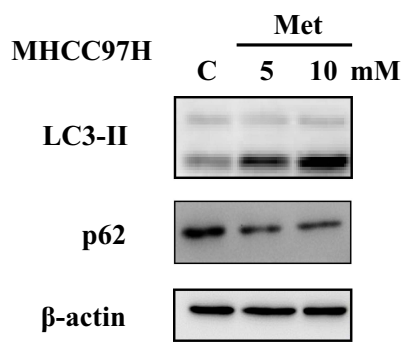

E HepG2 $\frac{12 \mathrm{~h}}{\mathrm{C} \text { Met }} \frac{24 \mathrm{~h}}{\mathrm{C} \text { Met }} \frac{48 \mathrm{~h}}{\mathrm{C} \text { Met }} \frac{72 \mathrm{~h}}{\mathrm{C} \text { Met STS }}$

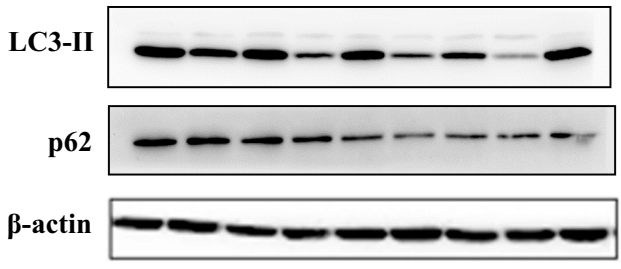

Figure 2 EGFP-LC3 plasmid transfection and metformin-induced autophagy in MHCC97H cells. (A and B) MHCC97H cells were transfected with a EGFP-LC3 plasmid. After incubation for $24 \mathrm{~h}$, they were treated without or with metformin (5 and $10 \mathrm{mM}$ ) for $48 \mathrm{~h}$ and were visualized with the confocal laser scanning microscope (original magnification $\times 63$, scale bar, $20 \mu \mathrm{m}$ ). The representative column diagrams showed the results and data were expressed as means \pm standard deviation. (C and $\mathbf{D})$ MHCC97H cells were treated with metformin $(10 \mathrm{mM})$ for $12,24,48$, and $72 \mathrm{~h}(\mathbf{C})$, and treated with metformin at various concentrations $(5$ and $10 \mathrm{mM})$ for $48 \mathrm{~h}(\mathbf{D})$. Expression of LC3-II and p62 in MHCC97H cells was detected by Western blot analysis. (E) HepG2 HCC cells were treated with metformin (I0 mM) for I2, 24, 48, and 72 h, and LC3-II and $\mathrm{p} 62$ was detected by Western blot analysis. The "C" indicates the control group, the "Met" indicates the metformin-treated groups, and the "STS" indicates the group which is treated with staurosporine. All the experiments were repeated thrice.

Abbreviations: C, control; h, hour; Met, metformin; STS, staurosporine.

significantly after treatment with metformin, indicating that AMPK expression was activated.

\section{Metformin Reduced the Phosphorylation Levels of mTOR and P70 S6 Kinase}

mTOR regulates cell growth and autophagy, and cell autophagy is inhibited by mTOR expression. ${ }^{12}$ MHCC97H cells were treated with metformin at the above-mentioned concentrations for $72 \mathrm{~h}$ and the expression levels of phosphoAMPK $\alpha$ (Thr172), phospho-mTOR (Ser2448), mTOR, phospho-p70 S6 Kinase (Thr421/Ser424) and p70 S6 Kinase were measured by Western blot (Figure 3B). The results showed that, with the enhancement of AMPK activity, treatment with metformin could significantly reduce the phosphorylation levels of mTOR and p70 S6 Kinase expression.

\section{Autophagy Inhibitor 3-MA Partly Abolished Metformin-Induced Autophagic Flux}

Ubiquitin-associated protein p62 is usually used to monitor the autophagic flux. ${ }^{18}$ An increased level of p62 expression indicates that the autophagic flux is inhibited, whereas a decreased level indicates that the autophagic flux is 


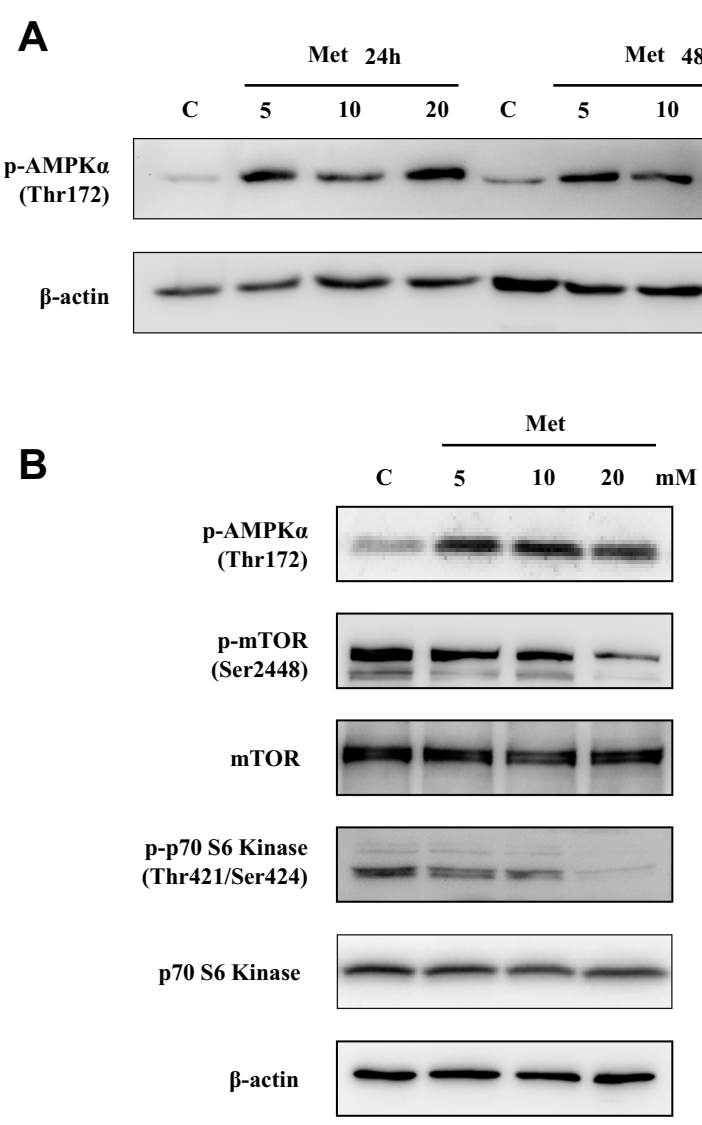

Figure 3 Metformin induces autophagy via the AMPK-mTOR signaling pathway in MHCC97H cells. (A) MHCC97H cells were treated with metformin (5, 10 and $20 \mathrm{mM}$ ) for 24 and $48 \mathrm{~h}$. Cell lysates were separated by SDS-PAGE and the phosphorylation level of AMPK was analyzed by Western blot. (B) MHCC97H cells were treated with metformin at the above-mentioned concentrations for $72 \mathrm{~h}$ and the expression levels of phospho-AMPK $\alpha$ (Thrl72), phospho-mTOR (Ser2448), mTOR, phospho-p70 S6 Kinase (Thr42I/Ser424) and p70 S6 Kinase were measured by Western blot. The "C" indicates the control group without treatment, and the "Met" indicates the groups treated with metformin. All the experiments were repeated thrice.

Abbreviations: C, control; h, hour; Met, metformin.

induced. As shown in Figure 2C-E, metformin could lead to decreased level of protein $\mathrm{p} 62$ in a dose- and time-dependent manner, indicating that metformin could induce the autophagic flux in MHCC97H and HepG2 cells. To further investigate metformin and autophagic flux, autophagy inhibitor 3-methyladenine (3-MA) was used and MHCC97H cells were pretreated with 3-MA $(10 \mathrm{mM})$ for $2 \mathrm{~h}$ (Figure $4 \mathrm{~A})$. Compared with the expression level of p62 simply treated with metformin, the combination of 3-MA and metformin could lead to the increased level of p62 and decreased level of LC3-II expression.

\section{mCherry-GFP-LC3B Plasmid Transfection and Metformin-Induced Autophagy}

MHCC97H cells were transfected with a mCherry-GFPLC3B plasmid to further study the effect of metformin on the autophagic flux (Figure 4B). Two fluorescent signals could be observed with the confocal microscopy. Yellow signals indicate the change of autophagosome and red signals represent the change of autolysosome. Increasing level of both yellow and red signals indicates that autophagic flux is induced. We found that treatment with metformin could induce the autophagic flux.

\section{Discussion}

Metformin is one widely used first-line anti-hyperglycemic drug and abundant evidences have demonstrated that metformin treatment can reduce the risk of some cancers. Considering the association of autophagy with the initiation and progression of cancers, our study was designed to determine the effect of metformin on the autophagy and autophagic flux via the AMPK-mTOR signaling pathway in human HCC cells. We found that metformin could induce the autophagy and autophagic flux, lead to the increased level of LC3-II and decreased level of p62, activate the AMPK expression, and inhibit the phosphorylation levels of mTOR and p70 S6 Kinase.

Our result that metformin could induce the autophagy of HCC cells was consistent with some previous published researches. ${ }^{19,20}$ Tsai et al found that metformin could activate autophagy in Huh7 cells and the underlying mechanisms in this process were suggested. ${ }^{19}$ They showed that metformin treatment led to a reduced degradation of Src-mediated CCAAT/enhancer-binding protein delta (CEBPD) protein and an increased level of CEBPDregulated LC3-II and ATG3 gene transcription. Another study was designed to determine the effect of combination of sorafenib and metformin on the autophagy via the mTOR pathway in HCC cells. ${ }^{20}$ The authors discovered that metformin could induce the activation of mTORC2 and suppress the mTORC1 and MAPK pathway, which may be responsible for the induction of autophagy.

However, there are still dissenting voices on the association of metformin with cell autophagy, for example, this following study which was designed to investigate the effect of metformin on autophagy and apoptosis in H4IIE rat HCC cells. ${ }^{15}$ The author found that metformin treatment could reduce the viability of H4IIE cells, stimulate the pro-apoptotic events (nuclear condensation, hydrolysis of intact poly ADP ribose polymerase and caspase-3), but decrease the expression levels of autophagy-related proteins (LC3B, beclin-1, Atg3, Atg5, Atg7, and Atg12). ${ }^{15}$ As a researcher, the author naturally knew that metformin had been reported to induce autophagy in various types of 
A

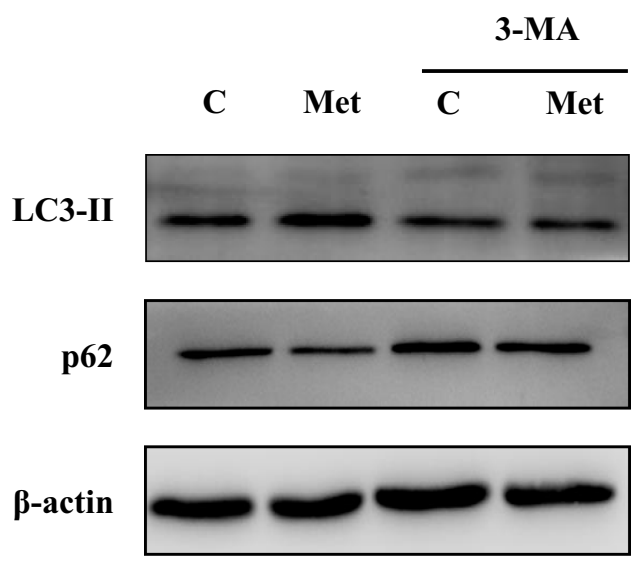

B

GFP
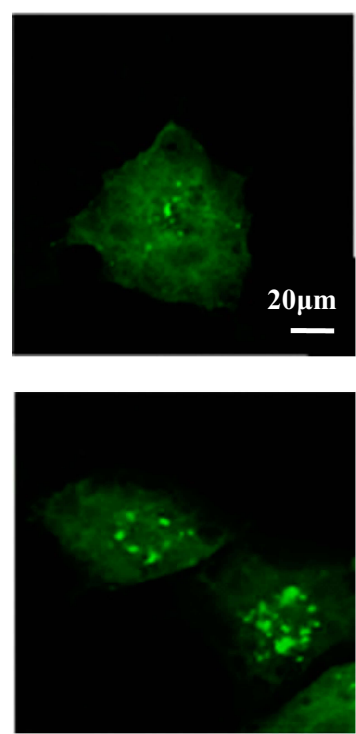

mCherry
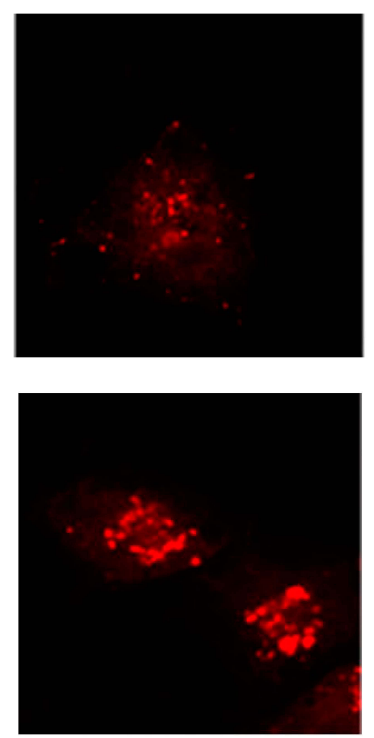

mCherry-GFP
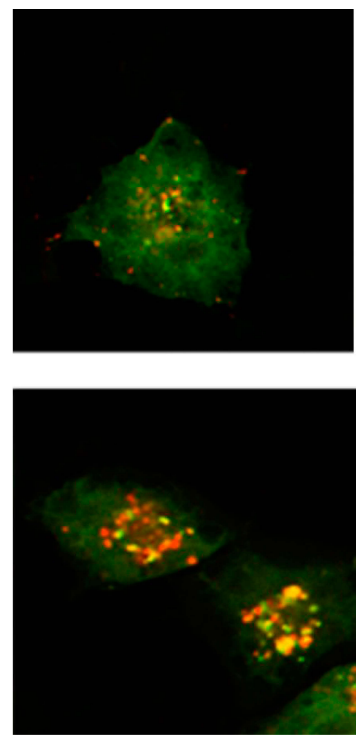

Figure 4 Autophagy inhibitor 3-methyladenine (3-MA) and mCherry-GFP- LC3B plasmid transfection in metformin-induced autophagic flux. (A) MHCC97H cells were pretreated with 3-MA (10 mM) for $2 \mathrm{~h}$, followed by treatment with metformin (10 mM) for $48 \mathrm{~h}$. The LC3-II turnovers and p62 level were detected by Western blot. (B) MHCC97H cells were transfected with a mCherry-GFP-LC3B plasmid, followed by treatment with metformin (I0 mM) for $48 \mathrm{~h}$. Representative fluorescent images were visualized with confocal microscopy (original magnification $\times 63$, scale bar, $20 \mu \mathrm{m}$ ). Yellow signals indicate the change of autophagosome and red signals represent the change of autolysosome. Increasing level of both yellow and red signals simultaneously indicates that autophagic flux is induced in MHCC97H cells. The "C" or "Control" indicates the blank control group, and the "Met" indicates the groups treated with metformin. All the experiments were repeated thrice.

Abbreviations: C, control; Met, metformin; 3-MA, 3-methyladenine.

cells, ${ }^{15,21-23}$ and as described in the section of discussion, the author declared that it was the first report showing that metformin inhibited autophagy in HCC cells. ${ }^{15}$

Coincidentally, as shown in Figure 2C-E of our study, we observed that metformin could decrease the expression level of LC3-II in HepG2 cells which was similar to the abovementioned report. However, it was increased in MHCC97H cells, and the p62 expression was decreased both in MHCC97H and HepG2 cells. LC3 is involved in the formation of autophagosomes and p62 is usually used to monitor the autophagic flux. Therefore, we deduced that metformin might have no obvious effect on the formation of autophagosomes in HepG2 cells, but it could affect the autophagy flux and induce the cell autophagy. In addition, it might be different in different types of HCC cells.

AMPK-mTOR signaling pathway is well known to be associated with autophagy and this pathway has been reported to be the potential mechanism for multiple molecular targets and therapeutic approaches in HCC, for example radiofrequency ablation (RFA) and heat stress, bifunctional enzyme ATIC, glycochenodeoxycholate (GCDC) and SOX18 expression. ${ }^{24-27}$ RFA and heat 
stress could induce the autophagy in HCC SMMC7721 and Huh7 cells, and autophagy was induced via the ATPAMPK-mTOR axis. $^{26} \mathrm{Li}$ et al demonstrated that ATIC expression was up-regulated in $\mathrm{HCC}$ tissues and $\mathrm{HCC}$ patients with high level of ATIC had poor survival. They declared that ATIC was identified as one oncogenic gene that promotes proliferation and migration vis the AMPKmTOR-S6 K1 signaling. ${ }^{24}$ GCDC and downregulation of SOX18 could also activate the autophagy by targeting the AMPK-mTOR pathway in HCC cells. ${ }^{25,27}$

Some limitations should be acknowledged in our study. The first was that MHCC97H and HepG2 cell lines were used and the reported experiment results were mostly from the MHCC97H cells considering LC3-II expression was decreased by metformin in HepG2 cells. We hope that these results can be validated in more HCC cell lines. The second was that only some autophagy-related proteins and AMPK-mTOR signaling pathways were examined, and the results were only from in vitro cell culture tests. We wish that more signaling pathways and molecular biomarkers could be verified in future studies, and more experimental methods and research strategies are adopted.

In conclusion, our study found that metformin treatment could induce the autophagy, autophagic flux, and activate the AMPK-mTOR signaling pathway in human HCC cells. We anticipate that combination treatment with metformin and molecular markers of the AMPK-mTOR signaling pathway could be used in HCC therapy.

\section{Ethics Approval}

Our research project was approved by the Clinical Research Ethics Committee of China-Japan Friendship hospital (Beijing, China). The human HCC HepG2 cell lines had been authenticated by STR profile and the use was approved by the Clinical Research Ethics Committee of China-Japan Friendship hospital.

\section{Author Contributions}

All authors contributed to data analysis, drafting or revising the article, gave final approval of the version to be published, and agree to be accountable for all aspects of the work.

\section{Funding}

This work was supported by Z131107000413067 from the Beijing NOVA Programme of Beijing Municipal Science \& Technology Commission.

\section{Disclosure}

The authors declare that they have no conflicts of interest.

\section{References}

1. Siegel RL, Miller KD, Jemal A. Cancer statistics, 2019. CA Cancer J Clin. 2019;69(1):7-34. doi:10.3322/caac.21551

2. Gao C. Molecular pathological epidemiology in diabetes mellitus and risk of hepatocellular carcinoma. World J Hepatol. 2016;8 (27):1119-1127. doi:10.4254/wjh.v8.i27.1119

3. Zhang H, Gao C, Fang L, Zhao HC, Yao SK. Metformin and reduced risk of hepatocellular carcinoma in diabetic patients: a meta-analysis. Scand J Gastroenterol. 2013;48(1):78-87. doi:10.3109/00365521. 2012.719926

4. Gao C. Molecular pathological epidemiology: an interdisciplinary field for study of hepatocellular carcinoma. Austin J Gastroenterol. 2015;2(3):1040.

5. Umetsu S, Mizukami H, Saito T, et al. Diabetes, an independent poor prognostic factor of non-B non-C hepatocellular carcinoma, correlates with dihydropyrimidinase-like 3 promoter methylation. Sci Rep. 2020;10(1):1156. doi:10.1038/s41598-020-57883-1

6. Luo X, Sui J, Yang W, et al. Type 2 diabetes prevention diet and hepatocellular carcinoma risk in US men and women. $\mathrm{Am}$ $J$ Gastroenterol. 2019;114(12):1870-1877. doi:10.14309/ajg.000 0000000000450

7. Chen X, Zhang F, Li J, et al. Tai Chi for type 2 diabetes mellitus: a protocol for systematic review. Medicine. 2020;99(4):e18853. doi:10.1097/MD.0000000000018853

8. Kamarudin MNA, Sarker MMR, Zhou JR, Parhar I. Metformin in colorectal cancer: molecular mechanism, preclinical and clinical aspects. J Exp Clin Cancer Res. 2019;38(1):491. doi:10.1186/ s13046-019-1495-2

9. Lord SR, Collins JM, Cheng WC, et al. Transcriptomic analysis of human primary breast cancer identifies fatty acid oxidation as a target for metformin. Br J Cancer. 2020;122(2):258-265. doi:10.1038/ s41416-019-0665-5

10. Zhang X, Liu P, Shang Y, et al. Metformin and LW6 impairs pancreatic cancer cells and reduces nuclear localization of YAP1. J Cancer. 2020;11(2):479-487. doi:10.7150/jca.33029

11. Li X, He S, Ma B. Autophagy and autophagy-related proteins in cancer. Mol Cancer. 2020;19(1):12. doi:10.1186/s12943-020-1138-4

12. Xi X, Zou C, Ye Z, et al. Pioglitazone protects tubular cells against hypoxia/reoxygenation injury through enhancing autophagy via AMPK-mTOR signaling pathway. Eur J Pharmacol. 2019;863:172695. doi:10.1016/j.ejphar.2019.172695

13. Fan H, Yu X, Zou Z, et al. Metformin suppresses the esophageal carcinogenesis in rats treated with NMBzA through inhibiting AMPK/ mTOR signaling pathway. Carcinogenesis. 2019;40(5):669-679. doi:10.1093/carcin/bgy160

14. Abdelmonsif DA, Sultan AS, El-Hadidy WF, Abdallah DM, Targeting AMPK. mTOR and beta-catenin by combined metformin and aspirin therapy in HCC: an appraisal in Egyptian HCC patients. Mol Diagn Ther. 2018;22(1):115-127. doi:10.1007/s40291-0170307-7

15. Park DB. Metformin promotes apoptosis but suppresses autophagy in glucose-deprived H4IIE hepatocellular carcinoma cells. Diabetes Metab J. 2015;39(6):518-527. doi:10.4093/dmj.2015.39.6.518

16. Wang L, Gao C, Yao S, Xie B. Blocking autophagic flux enhances matrine-induced apoptosis in human hepatoma cells. Int J Mol Sci. 2013;14(12):23212-23230. doi:10.3390/ijms 141223212

17. Wu YY, Zheng BR, Chen WZ, et al. Expression and role of autophagy related protein $\mathrm{p} 62$ and LC3 in the retina in a rat model of acute ocular hypertension. Int $J$ Ophthalmol. 2020;13(1):21-28. doi:10. 18240/ijo.2020.01.04 
18. Xu XW, Pan CW, Yang XM, et al. SP1 reduces autophagic flux through activating p62 in gastric cancer cells. Mol Med Rep. 2018;17(3):4633-4638. doi:10.3892/mmr.2018.8400

19. Tsai HH, Lai HY, Chen YC, et al. Metformin promotes apoptosis in hepatocellular carcinoma through the CEBPD-induced autophagy pathway. Oncotarget. 2017;8(8):13832-13845. doi:10.18632/oncotarget. 14640

20. Ling S, Song L, Fan N, et al. Combination of metformin and sorafenib suppresses proliferation and induces autophagy of hepatocellular carcinoma via targeting the mTOR pathway. Int $J$ Oncol. 2017;50(1):297-309. doi:10.3892/ijo.2016.3799

21. Jiang Y, Huang W, Wang J, et al. Metformin plays a dual role in MIN6 pancreatic beta cell function through AMPK-dependent autophagy. Int J Biol Sci. 2014;10(3):268-277. doi:10.7150/ijbs.7929

22. Jiang T, Yu JT, Zhu XC, et al. Acute metformin preconditioning confers neuroprotection against focal cerebral ischaemia by pre-activation of AMPK-dependent autophagy. $\mathrm{Br} J$ Pharmacol. 2014;171(13):3146-3157. doi:10.1111/bph.12655

23. He C, Zhu H, Li H, Zou MH, Xie Z. Dissociation of Bcl-2-Beclin1 complex by activated AMPK enhances cardiac autophagy and protects against cardiomyocyte apoptosis in diabetes. Diabetes. 2013;62 (4):1270-1281. doi:10.2337/db12-0533
24. Li $\mathrm{M}$, Jin $\mathrm{C}, \mathrm{Xu} \mathrm{M}$, et al. Bifunctional enzyme ATIC promotes propagation of hepatocellular carcinoma by regulating AMPKmTOR-S6 K1 signaling. Cell Commun Signal. 2017;15(1):52. doi:10.1186/s12964-017-0208-8

25. Gao L, Lv G, Li R, et al. Glycochenodeoxycholate promotes hepatocellular carcinoma invasion and migration by AMPK/mTOR dependent autophagy activation. Cancer Lett. 2019;454:215-223. doi:10.1016/j.canlet.2019.04.009

26. Jiang J, Chen S, Li K, et al. Targeting autophagy enhances heat stress-induced apoptosis via the ATP-AMPK-mTOR axis for hepatocellular carcinoma. Int $J$ Hyperthermia. 2019;36(1):499-510. doi:10.1080/02656736.2019.1600052

27. Sun Y, Lei B, Huang Q. SOX18 affects cell viability, migration, invasiveness, and apoptosis in Hepatocellular Carcinoma (HCC) cells by participating in Epithelial-to-Mesenchymal Transition (EMT) progression and Adenosine Monophosphate Activated Protein Kinase (AMPK)/Mammalian Target of Rapamycin (mTOR). Med Sci Monit. 2019;25:6244-6254. doi:10.12659/MSM.915729

\section{Publish your work in this journal}

Cancer Management and Research is an international, peer-reviewed open access journal focusing on cancer research and the optimal use of preventative and integrated treatment interventions to achieve improved outcomes, enhanced survival and quality of life for the cancer patient.
The manuscript management system is completely online and includes a very quick and fair peer-review system, which is all easy to use. Visit http://www.dovepress.com/testimonials.php to read real quotes from published authors. 